\title{
The Axolotl Model for Regeneration and Aging Research: A Mini-Review
}

\author{
Catherine McCusker David M. Gardiner \\ Department of Developmental and Cell Biology, and Developmental Biology Center, University of California Irvine, \\ Irvine, Calif., USA
}

\author{
Key Words \\ Axolotl · Urodele $\cdot$ Regeneration · Fibroblasts · Aging • \\ Rejuvenation
}

\begin{abstract}
Although regeneration of tissues occurs in all adult tetrapods, the ability to regenerate complex structures such as limbs is limited to urodeles (newts and salamanders). Given that many of the biological processes and the signaling pathways that control these processes are highly conserved among all tetrapods, it is likely that humans have the potential to regenerate structures in the same way as salamanders. Thus the remarkable regenerative abilities of salamanders demonstrate what we reasonably can expect in terms of enhancing our regenerative potential. Although most of what is understood about regenerative mechanisms pertains to the repair of acute injuries, we assume that these same mechanisms could be utilized therapeutically to slow or even reverse chronic damage associated with aging. The axolotl model provides the opportunity to understand the behavior of cells to give the desired outcome of controlled growth and pattern formation leading to regeneration rather than aging and cancer. In this paper we present an overview of several important aspects of regeneration biology with an emphasis on the Mexican axolotl (Ambystoma mexicanum) as a model organism for identifying relevant signaling pathways and factors regulating limb regeneration. We also speculate about how these mechanisms could be utilized to reverse the aging process. By understanding the
\end{abstract}

mechanisms of regeneration, we eventually will be able to enhance our intrinsic regenerative abilities in order to slow and even reverse the damage of aging.

$$
\text { Copyright } \odot 2011 \text { S. Karger AG, Basel }
$$

\section{Introduction}

Life requires the maintenance of a balance between the processes that damage our tissues and the regenerative mechanisms that repair that damage. As we age, degenerative processes such as acute and chronic injuries continue to occur while we gradually lose our regenerative abilities. It is the combination of these 2 features over time that results in the loss of vitality and the transition to frailty that we call aging. It follows from the point of view of a regeneration biologist that both enhancing what we can regenerate and reversing the age-associated decline in that ability is the road forward in terms of extending our life/health span. Therefore, understanding the mechanisms of the regenerative abilities that we already have, along with developing strategies to activate and enhance this potential, are essential to maintaining or restoring a more youthful balance.

In response to acute injury, we are able to repair tissues such as bone, peripheral nerves and epithelial tissues. We also are able to repair chronic damage, primarily to epithelial tissues exposed to environmental insult, such as excessive exposure to sunlight. In addition, the integrity of many of our tissues over time is dependent on a con-

\section{KARGER \\ Fax +41613061234 \\ E-Mail karger@karger.ch}

www.karger.com
(C) 2011 S. Karger AG, Basel

0304-324X/11/0576-0565\$38.00/0

Accessible online at:

www.karger.com/ger
Dr. David M. Gardiner

Department of Developmental and Cell Biology, Natural Sciences II Division

University of California Irvine, 4111 Natural Sciences II

Irvine, CA 92697-2305 (USA)

Tel. +1 949824 2792, E-Mail dmgardin@ uci.edu 
tinuous renewal process involving replacement of lost cells by adult stem cells, such as blood cells and the intestinal epithelium (see [1]). This balance between loss and replacement is critical to maintaining tissue structure and function. The ability to replace cells within a specific tissue, though impressive, is limited and, with few exceptions (e.g. finger tips [2]), we do not regenerate more complex structures that integrate the structure and function of multiple tissues. In contrast, one group of tetrapods, the urodele amphibians (newts and salamanders) do have the ability to regenerate complex structures such as limbs and spinal cords. The remarkable regenerative abilities of salamanders demonstrate what we reasonably can expect in terms of enhancing our regenerative potential. While the ability of these animals to regenerate entire organs seems extraordinary, the mechanisms of regeneration involve many basic biological processes regulated by conserved signaling pathways [3]. Thus, we can expect to stimulate regeneration in a human by the same mechanisms used by the axolotl. In this review, we present an overview of the Mexican axolotl (Ambystoma mexicanum) as a model organism for identifying the relevant signaling pathways and factors regulating limb regeneration, and speculate about how to enhance our regenerative responses to both acute injury and the accumulation of chronic damage to slow or even reverse the loss of both tissue and organ function associated with aging.

\section{Mechanisms of Regeneration in Response to Acute Injury}

Historically, studies of regeneration have focused on either animals that can regenerate (e.g. axolotl) or those that cannot (e.g. mouse). In the latter case, the strategy typically has been to test factors and treatments to induce regeneration, and in the former to describe the cellular and molecular events associated with regeneration with the goal of understanding the mechanisms regulating regeneration. Neither approach has led to the anticipated and desired breakthroughs in terms of inducing regeneration in humans. Given that successful regeneration likely involves a complex sequence of events, it is not surprising that attempts to induce regeneration in a nonregenerating animal with a single factor or treatment have been unsuccessful. Ironically, studying animals that can regenerate also has failed to provide useful insights into potential therapies. Because these animals always regenerate, it is possible to describe how regeneration happens, but it is not possible to test whether or not a candidate factor can induce a regenerative response. Consequently, experimental studies in animals such as salamanders and fish have been designed as loss-of-function experiments in which regeneration is first inhibited, and then candidate regeneration-inducting factors are tested to determine whether or not they can rescue regeneration. This approach has identified factors and biological processes that are required for regeneration to occur (e.g. cellular proliferation), but have yet to identify signals that induce regeneration in a normally non regenerating wound.

Given the limitations of the loss-of-function approach to studying regeneration, we have focused our efforts in recent years to developing gain-of-function assays for regeneration. One such approach has been to use the induction of ectopic blastemas (a population of undifferentiated regeneration-competent cells) and limbs as an assay for regeneration-inducing signaling pathways (The Accessory Limb Model [4]). This model is based on classic studies demonstrating that an entire limb can be induced to form on the side of the arm when appropriate signals from nerves and connective tissue fibroblasts are provided (fig. 1). In the absence of these signals, the wound heals but no ectopic structures are formed. This model thus reduces the complexity associated with amputations in which considerable damage is done to limb tissues, resulting in injury responses that may or may not be associated with signaling that induces regeneration. Injury is limited to the dermal connective tissue that is the source of the early blastema cells (discussed below), and the signals required to induce regeneration are provided by the deviated nerve and grafted skin (fig. 1). It thus is possible to test whether candidate factors can induce blastema formation and de novo limb formation.

The Accessory Limb Model emphasizes the progression of regeneration as a stepwise process that is initiated by signals associated with injury leading eventually to regeneration of a new limb. The initial event is re-epithelialization to cover the wound surface, and the wound heals. If additional signals from the nerve are provided, dermal fibroblasts at the wound periphery are induced to dedifferentiate, migrate toward the nerve and aggregate to form the early blastema. Finally, if dermal cells from the opposite side of the limb are grafted to the wound site, the ectopic blastema continues to the next steps in the regeneration pathway and a new limb is formed. One important point is that regeneration-competent cells will fail to form a regenerate unless all of these criteria are met. The challenge is to identify each step and determine which steps are the barriers to regenerative success [2]. In the context of the decline in regenerative abilities with age, it is possible 
Fig. 1. The induction of ectopic blastemas and limbs in the axolotl (Ambystoma mexicanum). A full-thickness skin wound on the side of the arm heals without forming ectopic structures. a If the brachial nerve is dissected free and severed, the end can be deviated surgically to the wound site on the side of the arm (small square). About 5-7 days later, an ectopic blastema forms (c), but it does not form a new limb. b If in addition to the wound and deviated nerve, a piece of skin from the opposite side of the limb is grafted to the wound site (posterior graft to anterior wound is illustrated), a normally patterned ectopic limb is induced to form (d). (After Bryant et al. [3].)

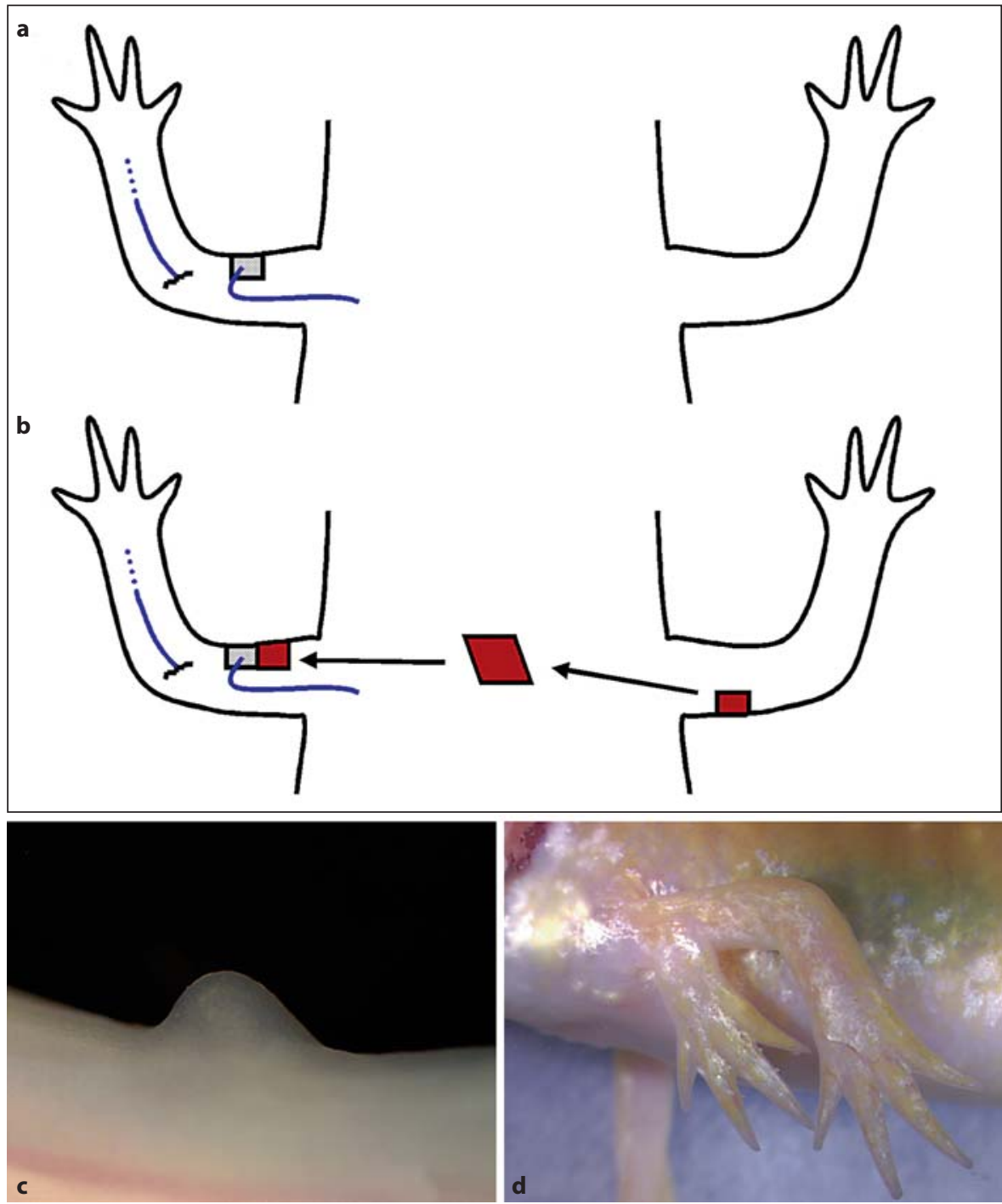

that not all the steps are vulnerable to the effects of aging and that there is a progressive decline at only one or a few critical steps. Thus identification of such barriers will identify the targets for therapeutic interventions to enhance our regenerative abilities in both youth and old age.

\section{Regeneration and Aging in Amphibians}

Although much is known about the mechanisms of regeneration in salamanders, essentially nothing is known about aging in these animals. Therefore, an unanswered question is whether regenerating animals such as the axolotl age the same way as nonregenerating animals such as humans. For example, do axolotl tissues ac- cumulate damage and become progressively less functional over the chronological age of the animal? As with other animals, we can know the chronological age of an axolotl, but since biomarkers for aging are limited, we cannot determine the extent to which the animal has aged, i.e. its biological age. A second key question is whether regenerated tissues are the same biological age as the rest of the animal or whether they become young again during regeneration.

It appears that the ability to regenerate developing organs such as limb buds and the spinal cord occurs in many, if not all vertebrates while they are embryos (see [5]). As the embryo develops, this impressive regenerative ability is progressively lost, and by birth it is gone [5]. Adult salamanders are unique in that the ability to regen- 
erate an organ persists beyond the embryonic/larval period. As an aside, it has been noted that axolotls are neotenous and therefore never complete metamorphosis. Although one might consider there to be a relationship between the retention of some larval characteristics and the ability to regenerate, that appears not to be the case. Postmetamorphic axolotls that have completed metamorphosis (either spontaneously or by experimental treatment with thyroid hormone) as well as postmetamorphic newts respond to injury and regenerate their limbs like neotenous axolotls. Therefore neotenous axolotls, like other salamanders, are postembryonic, the tissues of their organs (e.g. limbs) are differentiated, and their capacity to regenerate is a consequence of the ability to reaccess the regenerative program of the embryo. Thus the ontogenetic decline and eventual loss of organ regeneration in other vertebrates is presumed to be a consequence of not being able to revert to the earlier state of limb development [3]. Although the capability to regenerate persists in the axolotl, it is widely recognized that it takes older animals longer to regenerate an arm than it does younger animals. This phenomenon of a decrease in the rate of regeneration with age has not been studied critically, and no mechanistic hypotheses have been proposed or tested to date. Finger tips in mammals, including humans, regenerate postnatally. Though this ability persists into adulthood, the rate and fidelity of regeneration appear to decline with age (see [6]). Again, this phenomenon has not been investigated quantitatively.

The ontogenetic decline and eventual loss in the ability to regenerate limb buds as embryos is progressive and is intrinsic to the cells of the limb bud [5]. Importantly, this loss can be delayed experimentally, suggesting that the loss of regenerative ability during aging may also be delayed or reversed. The loss of embryonic regenerative ability has been best studied in the larvae of the frog Xenopus laevis, in which early limb buds regenerate perfectly when amputated. Once the Xenopus limb has completed tissue differentiation, limb amputations result in the formation of a cartilaginous spike instead of a properly patterned limb [7]. At intermediate stages, regenerative ability is lost in a proximal to distal progression such that an amputation at a more distal level will still regenerate even though a more proximal level amputation fails to regenerate [7].

The loss of regenerative ability is intrinsic to the cells of the Xenopus limb bud such that a young limb bud grafted to an older larva regenerates, while a young larval host does not rescue the regenerative ability of a limb bud grafted from an older larva (see [5]). This intrinsic regenerative response can be enhanced by increased levels of fibroplast growth factor (FGF) signaling during the later stages of regenerative decline, but once the cells lose the ability to regenerate, FGF treatment is ineffective (see [5]). Finally, the period of regeneration competence in Xenopus can be extended by regeneration itself. A limb bud that is repeatedly reamputated is maintained in a continuously regenerating state long after the animal reaches the stage when regenerative abilities are normally lost in an unamputated limb (see [5]). Thus it is possible to override the signals that cause the loss of regenerative ability as the larva ages, indicating the feasibility for therapeutic interventions to enhance our intrinsic regenerative abilities to delay or even reverse damage associated with aging.

As to the question of whether regenerated tissues are young or old, casual observations would suggest that regenerated tissues are rejuvenated. It is evident that regenerated skin is morphologically distinct from old stump skin that is thicker and has more dermal connective tissue. Although the overall structure of the regenerated skin is characteristic of the less complex skin of younger animals, the cells within this tissue still may be the same biological age as those in the adjacent, old skin. Again, the issue of cellular aging in the axolotl has not been studied systematically (e.g. telomere length), and none of the limited biomarkers for aging skin and extracellular matrix (ECM) (e.g. advanced glycation endproducts) have been looked at. We note that regenerated arms can be reamputated multiple times, and that they continue to regenerate. Therefore, even if regenerated tissues are not rejuvenated, they nevertheless are capable of supporting regeneration. Finally, as noted above, regenerating limbs appear to revert to an embryonic-like developmental state followed by the recapitulation of the limb development program. It therefore is reasonable to hypothesize that regenerating tissues are embryonic-like and give rise to postembryonic tissues that are equivalent to the young tissues that originally developed at the end of embryogenesis.

Induced regeneration of cartilage in the axolotl [8] provides direct evidence that is consistent with the hypothesis that regenerated tissues are more like young tissue than old tissue. When a segment of the radius in the arm of an axolotl is surgically removed, it is not regenerated even though the entire arm will regenerate if amputated. The persistence of this deletion (critical size defect) is comparable to what happens in humans when the ends of the injured skeleton undergo a limited regenerative response and form a cartilaginous callus (see [8]). As in humans, the response to injury to the cartilage is formation of fibrocartilage, which is equivalent to the scarring response of injured connective tissue (discussed below). However, 
if the cells at the site of the injury in the axolotl are induced to undergo dedifferentiation and blastema cell formation, new hyaline cartilage forms and becomes integrated into the cut end of the bone. This regenerative response is induced by signaling between nerves and the wound epithelium (WE) that forms to cover the critical size defect, and is comparable to the response that is induced by amputation. Thus the endogenous response of the adult tissues (callus formation) in an old environment can be overridden by signaling from the nerve/WE to yield the outcome of embryonic tissues in a young environment. It is unknown whether the apparently rejuvenated tissues age at a normal rate or if aging is accelerated by factors in the old animal environment as reported for regeneration of muscle tissue [9]. Regardless, the results from this and a number of studies of regeneration in salamanders emphasize the importance of signaling from the nerve in the regulation of the developmental state of adult cells.

\section{Fibroblasts Are the Target for Regeneration and Rejuvenation Therapies}

One important understanding with regards to regeneration is that cells in the loose connective tissues are the progenitors of the blastema cells that control growth and pattern formation $[3,10,11]$. Although progenitor cells for the other limb tissues eventually enter the growing blastema and contribute to the other tissue types of the regenerated limb, the earliest blastema cells are derived from connective tissue fibroblasts. For the nonconnective tissues, the progenitor cells are lineage restricted, although the possibility of transdifferentiation during regeneration has repeatedly been reported (see [12]). In general, these limb tissue progenitor cells function as adult stem cells. For example, Schwann cells arise from preexisting Schwann cells in the stump [10], and regenerated muscle arises from preexisting muscle tissue in the stump (see [10]). The origin of the myoprogenitor cells during salamander limb regeneration is somewhat controversial at this point, and there appear to be 2 sources of these cells. As in muscle tissues of all other vertebrates, salamander muscle has an adult stem cell, the satellite cell, which has been widely studied in a number of injury models as a source of cells for muscle regeneration $[3,9$, $13,14]$. In addition to satellite cells, newt myotubes undergo fragmentation to give rise to mononucleate cells that also participate in muscle regeneration (see $[10,12$, 14]). In contrast to the lineage-restricted cells of the other limb tissues, cells from the dermis contribute to all of the connective tissue derivatives, including loose connective tissue, ligaments, tendons and cartilage [3, 15]. Early studies of cell contribution to the blastema demonstrated that these cells make up on average $50 \%$ of the cells of the early to medium bud blastema, and are the cells that control the pattern of the structures that are regenerated [3, 11]. Given the heterogeneity and the poorly defined phenotype of fibroblasts (discussed below), the challenge is to identify the cell type within the loose connective tissue that is the blastema cell progenitor.

As discussed above, signaling between the nerves and the WE is critical for inducing and maintaining the blastema, and it is the fibroblasts that are the target cells of those signals. In response to injury, connective tissue fibroblasts within a few hundred micrometers of the wound are induced to dedifferentiate and migrate to form the blastema [16-18]. The fibroblast-derived blastema cells are functionally equivalent to the mesenchymal cells of the developing limb bud and control the eventual pattern of the other cell types that migrate into the blastema from the stump, e.g. myoprogenitor cells $[3,11,15]$. The ability of dermal fibroblasts to reform the limb pattern has been demonstrated most directly in X-irradiated limbs with grafts of nonirradiated limb skin. These limbs do not have muscle (contribution from myoprogenitor cells, whether from satellite cells or fragmented myotubes, is blocked by X-irradiation). Nevertheless, the skeletal pattern of the limb is regenerated, along with the ligaments, tendons and muscle fascia (the connective tissue component of the muscle) in the appropriate pattern relative to the regenerated skeleton. Thus these limbs regenerate a normally-patterned limb derived entirely from the grafted dermal fibroblasts in the absence of participation from myoprogenitor cells [15].

In spite of the critical role of fibroblasts in the regulation of regeneration, very little is known about the biology of fibroblasts in general and salamander fibroblasts in particular. From studies of regeneration, it is evident that fibroblasts are not simple, spindle-shaped 'glue' cells, but rather have detailed information about their position within their tissue of origin (see [3]). Thus fibroblasts from different parts of the body have different properties (e.g. when head or flank skin fibroblasts are grafted to replace the limb skin fibroblasts, limb regeneration is inhibited [19]. Although limb skin fibroblasts can support limb regeneration, cells from different positions around the limb circumference are different in their positional identities. In fact, regeneration of the entire limb requires the interactions of fibroblast-derived blastema cells from opposite sides of the limb (e.g. anterior and posterior) in order to 
regenerate the normal limb pattern [4]. We note that microarry studies have demonstrated that human fibroblasts also differ in their patterns of gene expression between different tissues and organs, as well as between different positions within an organ such as the limb [20]. Thus it appears that spatially complex positional identities is a property of both salamander and human fibroblasts $[3,20]$.

Insight into the complex behavior of fibroblasts is essential for understanding regeneration. Fibroblast-derived blastema cells behave differently as a consequence of signaling from the nerve/WE. In the absence of these signals, fibroblasts accumulate in the wound bed and produce new ECM that is subsequently remodeled to restore the normal skin anatomy [4]. In contrast, these same cells respond to signals from the WE/AEC (apical epithelial cap) to dedifferentiate and proliferate as blastema cells [4]. Signaling from the WE/AEC is mediated in part by FGF (see $[3,21]$ ), leading to sustained distal outgrowth, presumably by the same mechanism as in developing limb buds. Since this signaling is spatially restricted to the distal AEC [21], as the blastema elongates, cells at more proximal levels are no longer under the influence of the AEC, and begin to redifferentiate and regenerate the dermis [22]. AEC signaling appears to maintain distal blastema cells in an undifferentiated state, and once released from the influence of the AEC, cells begin to differentiate and reform the connective tissue scaffolding and associated tissues. Thus regeneration involves the spatial and temporal regulation of the behavior of fibroblasts in order to balance the proliferation of undifferentiated blastema cells with the differentiation of these cells to reform the connective tissues of the limb. In contrast, these cells that induce and regulate regeneration in the salamander respond to injury with fibrosis and scar formation in humans. The challenge is to understand and regulate the behaviors of these complex cells.

\section{Challenges for Advances in Aging Research}

The lessons learned from axolotl regeneration are that successful regeneration requires both structure (e.g. muscle, bone and connective tissues) and information for restoring the appropriate patterns of the structures (provided by fibroblast progenitor cells). Therefore, presumably fibroblasts also would be important in terms of in situ regeneration to repair chronic injury associated with aging. Studies of the signals that regulate fibroblast behavior in response to acute injury have identified the nerve and WE as the source of the early signals leading to
Table 1. The 15 leading causes of death in the USA in 2006

Diseases of the heart (heart disease)*

Malignant neoplasms (cancer)

Cerebrovascular diseases (stroke)

Chronic lower respiratory diseases*

Accidents (unintentional injuries)

Diabetes mellitus (diabetes)

Alzheimer's disease

Influenza and pneumonia

Nephritis, nephrotic syndrome and nephrosis (kidney disease)*

Septicemia

Intentional self-harm (suicide)

Chronic liver disease and cirrhosis*

Essential hypertension and hypertensive renal disease

(hypertension)

Parkinson's disease

Assault (homicide)

National Vital Statistics Reports, Vol. 57, No. 14, April 17, 2009.

* Chronic diseases that involve tissue fibrosis.

formation of the regeneration-competent blastema cells. Therefore, studies to identify and characterize these signaling pathways along with their downstream targets should lead to strategies to induce in situ regeneration to repair chronic damage.

Although little is known about the regulation of fibroblast behavior in vivo, it is clear that fibrosis is associated with many age-related pathologies. Aside from cancer or traumatic injuries, fibrotic diseases (indicated by an asterisk in table 1) are among the major causes of organ failure and age-related mortality. Thus aside from entailing the ability to induce regeneration, the ability to regulate fibroblast behavior would lead to therapies to slow the progression of aging-related fibrotic diseases.

In addition to fibrosis, cancer progression is also differentially regulated in animals that can regenerate. In a series of classic studies involving the injection of carcinogenic chemicals, it was discovered that salamanders develop tumors at a much lower frequency than animals that do not regenerate. Rather than tumor formation, the typical response when an outgrowth is induced is the formation of normally patterned, ectopic limbs similar to what is observed in response to a deviated nerve $[4,23]$. Given that activation of many of the same signaling pathways occurs in tumors as well as in developing and regenerating tissues, the microenvironment associated with the ability to regenerate presumably is able to control the behavior of the induced tumor cells so as to lead to dif- 
ferentiation of a normal limb pattern. Thus the axolotl model provides the opportunity to understand the behavior of cells to give the desired outcome of controlled growth and pattern formation leading to regeneration rather than aging and cancer.

\section{Conclusion and Outlook}

With advances in genetics and molecular biology, we anticipate a progressive increase in our understanding of the convergence of mechanisms regulating development, regeneration, cancer and aging. It is becoming increasingly evident that the same signaling pathways are involved in regulating each of these processes [24]. With recent advances in genomic resources, the axolotl provides a unique opportunity to study and eventually understand the regulation of all these processes in the same organism. Although much is known about the mechanisms of regeneration in the axolotl, essentially nothing is known about aging, and thus there is the opportunity to pioneer and develop new concepts for rejuvenation and anti-aging therapies. By understanding the mechanisms of regeneration, we eventually will be able to enhance our intrinsic regenerative abilities in order to slow and even reverse the damage of aging.

\section{Acknowledgements}

We wish to thank Dr. Susan Bryant (UC Irvine), Dr. Tetsuya Endo (Aichi Gakuin University), Akira Sato (Okayama University) and Dr. Ken Muneoka (Tulane University) for the many hours of discussion of the ideas presented in this mini-review. D.M.G. is a member of the Scientific Advisory Board of Organogensis, Inc.

\section{References}

1 Stocum DL: Regenerative Biology and Medicine. Burlington, Academic Press, 2006, pp 448.

-2 Han M, Yang X, Taylor G, Burdsal CA, Anderson RA, Muneoka K: Limb regeneration in higher vertebrates: developing a roadmap. Anat Rec B New Anat 2005;287:14-24.

- 3 Bryant SV, Endo T, Gardiner DM: Vertebrate limb regeneration and the origin of limb stem cells. Int J Dev Biol 2002;46:887-896.

4 Endo T, Bryant SV, Gardiner DM: A stepwise model system for limb regeneration. Dev Biol 2004;270:135-145.

5 Gardiner DM: Ontogenetic decline of regenerative ability and the stimulation of human regeneration. Rejuvenation Res 2005;8:141153.

-6 Muneoka K, Allan CH, Yang X, Lee J, Han $\mathrm{M}$ : Mammalian regeneration and regenerative medicine. Birth Defects Res C Embryo Today 2008;84:265-280.

-7 Muneoka K, Holler-Dinsmore G, Bryant SV: Intrinsic control of regenerative loss in Xenopus laevis limbs. J Exp Zool 1986;240:47-54.

$\checkmark 8$ Satoh A, Cummings GM, Bryant SV, Gardiner DM: Neurotrophic regulation of fibroblast dedifferentiation during limb skeletal regeneration in the axolotl (Ambystoma mexicanum). Dev Biol 2010;337:444-457.

$\checkmark 9$ Conboy IM, Conboy MJ, Wagers AJ, Girma ER, Weissman IL, Rando TA: Rejuvenation of aged progenitor cells by exposure to a young systemic environment. Nature 2005; 433:760-764
10 Kragl M, Knapp D, Nacu E, Khattak S, Maden M, Epperlein HH, Tanaka EM: Cells keep a memory of their tissue origin during axolotl limb regeneration. Nature 2009;460: 60-65.

11 Muneoka K, Fox W, Bryant SV: Cellular contribution from dermis and cartilage to the regenerating limb blastema in axolotls. Dev Biol 1986;116:256-260.

12 Morrison JI, Borg P, Simon A: Plasticity and recovery of skeletal muscle satellite cells during limb regeneration. FASEB J 2010;23: 750-756.

13 Cameron JA, Hilgers AR, Hinterberger TJ: Evidence that reserve cells are a source of regenerated adult newt muscle in vitro. Nature 1986;321:607-610.

14 Morrison JI, Loof S, He P, Simon A: Salamander limb regeneration involves the activation of a multipotent skeletal muscle satellite cell population. J Cell Biol 2006; 172:433440.

15 Lheureux E: The origin of tissues in the Xirradiated regenerating limb of the newt Pleurodeles waltilii; in Fallon JF, Caplan AI (eds): Limb Development and Regeneration, Part A. New York, Liss Inc, 1983, pp 455-465.

16 Gardiner DM, Blumberg B, Komine Y, Bryant SV: Regulation of HoxA expression in developing and regenerating axolotl limbs. Development 1995;121:1731-1741.

17 Gardiner DM, Muneoka K, Bryant SV: The migration of dermal cells during blastema formation in axolotls. Dev Biol 1986;118: 488-493.
18 Mchedlishvili L, Epperlein HH, Telzerow A, Tanaka EM: A clonal analysis of neural progenitors during axolotl spinal cord regeneration reveals evidence for both spatially restricted and multipotent progenitors. Development 2007;134:2083-2093.

19 Tank PW: The effect of nonlimb tissues on forelimb regeneration in the axolotl, Ambystoma mexicanum. J Exper Zool 1987;244: 409-423.

20 Rinn JL, Bondre C, Gladstone HB, Brown PO, Chang HY: Anatomic demarcation by positional variation in fibroblast gene expression programs. PLoS Genet 2006;2:e119.

21 Satoh A, Graham GM, Bryant SV, Gardiner DM: Neurotrophic regulation of epidermal dedifferentiation during wound healing and limb regeneration in the axolotl (Ambystoma mexicanum). Dev Biol 2008;319:321-335.

-22 Satoh A, Bryant SV, Gardiner DM: Regulation of dermal fibroblast dedifferentiation and redifferentiation during wound healing and limb regeneration in the axolotl. Dev Growth Differ 2008;50:743-754.

23 Breedis C: Induction of accessory limbs and of sarcoma in the newt (Triturus viridescens) with carcinogenic substances. Cancer Res 1952;12:861-866.

24 Beachy PA, Karhadkar SS, Berman DM: Tissue repair and stem cell renewal in carcinogenesis. Nature 2004;432:324-331. 